\title{
Interacting neural networks and cryptography
}

\author{
Wolfgang Kinzel ${ }^{1}$ and Ido Kanter ${ }^{2}$ \\ 1 Institute for Theoretical Physics and Astrophysics, Universität Würzburg, Am \\ Hubland, 97074 Würzburg, Germany \\ 2 Minerva Center and Department of Physics, Bar-Ilan University, 52100 \\ Ramat-Gan, Israel
}

\begin{abstract}
Two neural networks which are trained on their mutual output bits are analysed using methods of statistical physics. The exact solution of the dynamics of the two weight vectors shows a novel phenomenon: The networks synchronize to a state with identical time dependent weights. Extending the models to multilayer networks with discrete weights, it is shown how synchronization by mutual learning can be applied to secret key exchange over a public channel.
\end{abstract}

\section{Introduction}

Neural networks learn from examples. This concept has extensively been investigated using models and methods of statistical mechanics [1.,2]. A "teacher" network is presenting input/output pairs of high dimensional data, and a "student" network is being trained on these data. Training means, that synaptic weights adopt by simple rules to the input/output pairs.

When the networks - teacher as well as student - have $N$ weights, the training process needs of the order of $N$ examples to obtain generalization abilities. This means, that after the training phase the student has achieved some overlap to the teacher, their weight vectors are correlated. As a consequence, the student can classify an input pattern which does not belong to the training set. The average classification error decreases with the number of training examples.

Training can be performed in two different modes: Batch and on-line training. In the first case all examples are stored and used to minimize the total training error. In the second case only one new example is used per time step and then destroyed. Therefore on-line training may be considered as a dynamic process: at each time step the teacher creates a new example which the student uses to change its weights by a tiny amount. In fact, for random input vectors and in the limit $N \rightarrow \infty$, learning and generalization can be described by ordinary differential equations for a few order parameters [3].

On-line training is a dynamic process where the examples are generated by a static network - the teacher. The student tries to move towards the teacher. However, the student network itself can generate examples on which it is trained. When the output bit is moved to the shifted input sequence, the network generates a complex time series 顿. Such networks are called bit (for 
binary) or sequence (for continuous numbers) generators and have recently been studied in the context of time series prediction $[5]$.

This work on the dynamics of neural networks - learning from a static teacher or generating time series by self interaction - has motivated us to study the following problem: What happens if two neural networks learn from each other? In the following section an analytic solution is presented [6], which shows a novel phenomenon: synchronization by mutual learning. The biological consequences of this phenomenon are not explored, yet, but we found an interesting application in cryptography: secure generation of a secret key over a public channel.

In the field of cryptography, one is interested in methods to transmit secret messages between two partners A and B. An opponent $\mathrm{E}$ who is able to listen to the communication should not be able to recover the secret message.

Before 1976, all cryptographic methods had to rely on secret keys for encryption which were transmitted between $\mathrm{A}$ and $\mathrm{B}$ over a secret channel not accessible to any opponent. Such a common secret key can be used, for example, as a seed for a random bit generator by which the bit sequence of the message is added (modulo 2).

In 1976, however, Diffie and Hellmann found that a common secret key could be created over a public channel accessible to any opponent. This method is based on number theory: Given limited computer power, it is not possible to calculate the discrete logarithm of sufficiently large numbers [7].

Here we show how neural networks can produce a common secret key by exchanging bits over a public channel and by learning from each other.

\section{Dynamic transition to synchronization}

Here we study mutual learning of neural networks for a simple model system: Two perceptrons receive a common random input vector $\underline{x}$ and change their weights $\underline{w}$ according to their mutual bit $\sigma$, as sketched in Fig. 1. The output bit $\sigma$ of a single perceptron is given by the equation

$$
\sigma=\operatorname{sign}(\underline{w} \cdot \underline{x})
$$

$\underline{x}$ is an $N$-dimensional input vector with components which are drawn from a Gaussian with mean 0 and variance $1 . \underline{w}$ is a $N$-dimensional weight vector with continuous components which are normalized,

$$
\underline{w} \cdot \underline{w}=1
$$

The initial state is a random choice of the components $w_{i}^{A / B}, i=1, \ldots N$ for the two weight vectors $\underline{w}^{A}$ and $\underline{w}^{B}$. At each training step a common random input vector is presented to the two networks which generate two output bits $\sigma^{A}$ and $\sigma^{B}$ according to (11). Now the weight vectors are updated by the perceptron learning rule [3]: 


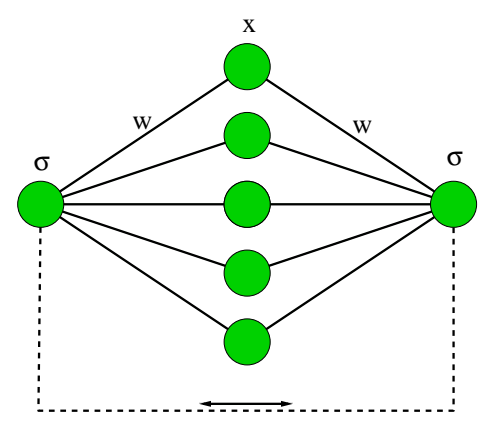

Fig. 1. Two perceptrons receive an identical input $\underline{x}$ and learn their mutual output bits $\sigma$.

$$
\begin{aligned}
& \underline{w}^{A}(t+1)=\underline{w}^{A}(t)+\frac{\eta}{N} \underline{x} \sigma^{B} \Theta\left(-\sigma^{A} \sigma^{B}\right) \\
& \underline{w}^{B}(t+1)=\underline{w}^{B}(t)+\frac{\eta}{N} \underline{x} \sigma^{A} \Theta\left(-\sigma^{A} \sigma^{B}\right)
\end{aligned}
$$

$\Theta(x)$ is the step function. Hence, only if the two perceptrons disagree a training step is performed with a learning rate $\eta$. After each step (3), the two weight vectors have to be normalized.

In the limit $N \rightarrow \infty$, the overlap

$$
R(t)=\underline{w}^{A}(t) \underline{w}^{B}(t)
$$

has been calculated analytically [6]. The number of training steps $t$ is scaled as $\alpha=t / N$, and $R(\alpha)$ follows the equation

$$
\frac{d R}{d \alpha}=(R+1)\left(\sqrt{\frac{2}{\pi}} \eta(1-R)-\eta^{2} \frac{\varphi}{\pi}\right)
$$

where $\varphi$ is the angle between the two weight vectors $\underline{w}^{A}$ and $\underline{w}^{B}$, i.e. $R=$ $\cos \varphi$. This equation has fixed points $R=1, R=-1$, and

$$
\frac{\eta}{\sqrt{2 \pi}}=\frac{1-\cos \varphi}{\varphi}
$$

Fig. 2 shows the attractive fixed point of 5 as a function of the learning rate $\eta$. For small values of $\eta$ the two networks relax to a state of a mutual agreement, $R \rightarrow 1$ for $\eta \rightarrow 0$. With increasing learning rate $\eta$ the angle between the two weight vectors increases up to $\varphi=133^{\circ}$ for

$$
\eta \rightarrow \eta_{c} \cong 1.816
$$

Above the critical rate $\eta_{c}$ the networks relax to a state of complete disagreement, $\varphi=180^{\circ}, R=-1$. The two weight vectors are antiparallel to each other, $\underline{w}^{A}=-\underline{w}^{B}$. 


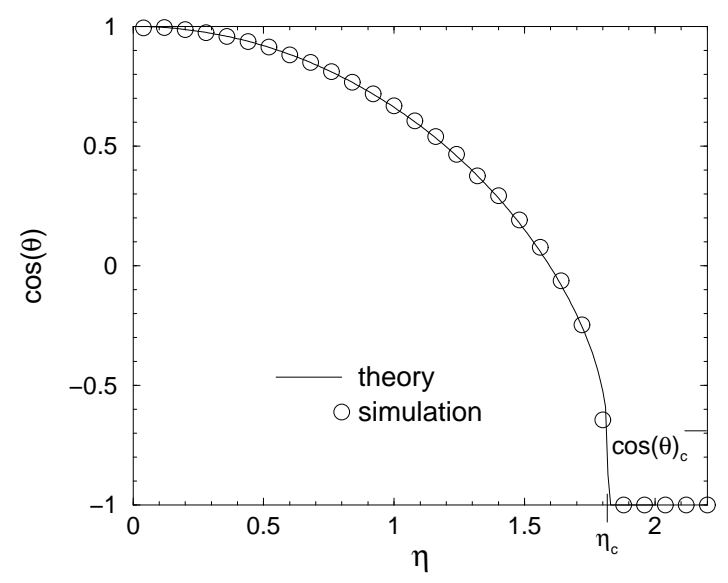

Fig. 2. Final overlap $R$ between two perceptrons as a function of learning rate $\eta$. Above a critical rate $\eta_{c}$ the time dependent networks are synchronized. From Ref. 6

As a consequence, the analytic solution shows, well supported by numerical simulations for $N=100$, that two neural networks can synchronize to each other by mutual learning. Both of the networks are trained to the examples generated by their partner and finally obtain an antiparallel alignment. Even after synchronization the networks keep moving, the motion is a kind of random walk on an $\mathrm{N}$-dimensional hypersphere producing a rather complex bit sequence of output bits $\sigma^{A}=-\sigma^{B}[8$.

\section{Random walk in weight space}

We want to apply synchronization of neural networks to cryptography. In the previous section we have seen that the weight vectors of two perceptrons learning from each other can synchronize. The new idea is to use the common weights $\underline{w}^{A}=-\underline{w}^{B}$ as a key for encryption [9]. But two problems have to be solved yet: (i) Can an external observer, recording the exchange of bits, calculate the final $\underline{w}^{A}(t)$, (ii) does this phenomenon exist for discrete weights? Point (i) is essential for cryptography, it will be discussed in the following section. Point (ii) is important for practical solutions since communication is usually based on bit sequences. It will be investigated in the following.

Synchronization occurs for normalized weights, unnormalized ones do not synchronize [6]. Therefore, for discrete weights, we introduce a restriction

in the space of possible vectors and limit the components $w_{i}^{A / B}$ to $2 L+1$ different values,

$$
w_{i}^{A / B} \in\{-L,-L+1, \ldots, L-1, L\}
$$

In order to obtain synchronization to a parallel - instead of an antiparallel state $\underline{w}^{A}=\underline{w}^{B}$, we modify the learning rule (3) to: 


$$
\begin{aligned}
& \underline{w}^{A}(t+1)=\underline{w}^{A}(t)-\underline{x} \sigma^{A} \Theta\left(\sigma^{A} \sigma^{B}\right) \\
& \underline{w}^{B}(t+1)=\underline{w}^{B}(t)-\underline{x} \sigma^{B} \Theta\left(\sigma^{A} \sigma^{B}\right)
\end{aligned}
$$

Now the components of the random input vector $\underline{x}$ are binary $x_{i} \in\{+1,-1\}$. If the two networks produce an identical output bit $\sigma^{A}=\sigma^{B}$, then their weights move one step in the direction of $-x_{i} \sigma^{A}$. But the weights should remain in the interval (8), therefore if any component moves out of this interval, $\left|w_{i}\right|=L+1$, it is set back to the boundary $w_{i}= \pm L$.

Each component of the weight vectors performs a kind of random walk with reflecting boundary. Two corresponding components $w_{i}^{A}$ and $w_{i}^{B}$ receive the same random number \pm 1 . After each hit at the boundary the distance $\left|w_{i}^{A}-w_{i}^{B}\right|$ is reduced until it has reached zero. For two perceptrons with a $N$-dimensional weight space we have two ensembles of $N$ random walks on the internal $\{-L, \ldots, L\}$. If we neglect the global signal $\sigma^{A}=\sigma^{B}$ as well as the bias $\sigma^{A}$, we expect that after some characteristic time scale $\tau=\mathcal{O}\left(L^{2}\right)$ the probability of two random walks being in different states decreases as

$$
P(t) \sim P(0) e^{-t / \tau}
$$

Hence the total synchronization time should be given by $N \cdot P(t) \simeq 1$ which gives

$$
t_{\text {sync }} \sim \tau \ln N
$$

In fact, our simulations for $N=100$ show that two perceptrons with $L=3$ synchronize in about 100 time steps and the synchronization time increases logarithmically with $N$. However, our simulations also showed that an opponent, recording the sequence of $\left(\sigma^{A}, \sigma^{B}, \underline{x}\right)_{t}$ is able to synchronize, too. Therefore, a single perceptron does not allow a generation of a secret key.

\section{Secret key generation}

Obviously, a single perceptron transmits too much information. An opponent, who knows the set of input/output pairs, can derive the weights of the two partners after synchronization. Therefore, one has to hide so much information, that the opponent cannot calculate the weights, but on the other side one has to transmit enough information that the two partners can synchronize.

In fact, we found that multilayer networks with hidden units may be candidates for such a task [9]. More precisely, we consider parity machines with three hidden units as shown in Fig. 3. Each hidden unit is a perceptron (11) with discrete weights (8). The output bit $\tau$ of the total network is the product of the three bits of the hidden units 


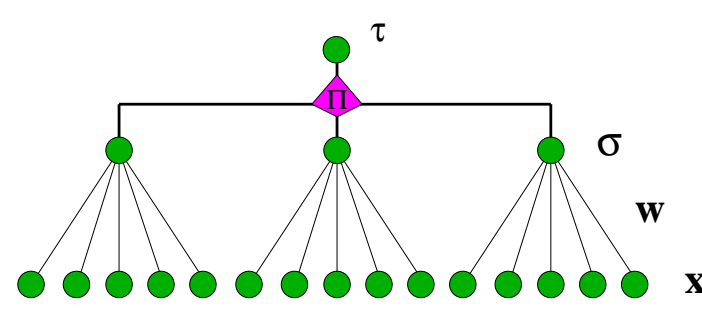

Fig. 3. Parity machine with three hidden units.

$$
\begin{aligned}
& \tau^{A}=\sigma_{1}^{A} \sigma_{2}^{A} \sigma_{3}^{A} \\
& \tau^{B}=\sigma_{1}^{B} \sigma_{2}^{B} \sigma_{3}^{B}
\end{aligned}
$$

At each training step the two machines $A$ and $B$ receive identical input vectors $\underline{x}_{1}, \underline{x}_{2}, \underline{x}_{3}$. The training algorithm is the following: Only if the two output bits are identical, $\tau^{A}=\tau^{B}$, the weights can be changed. In this case, only the hidden unit $\sigma_{i}$ which is identical to $\tau$ changes its weights using the Hebbian rule

$$
\underline{w}_{i}^{A}(t+1)=\underline{w}_{i}^{A}(t)-\underline{x}_{i} \tau^{A}
$$

For example, if $\tau^{A}=\tau^{B}=1$ there are four possible configurations of the hidden units in each network:

$$
(+1,+1,+1),(+1,-1,-1),(-1,+1,+1),(-1,-1,+1)
$$

In the first case, all three weight vectors $\underline{w}_{i}, \underline{w}_{2}, \underline{w}_{3}$ are changed, in all other three cases only one weight vector is changed. The partner as well as any opponent does not know which one of the weight vectors is updated.

The partners $A$ and $B$ react to their mutual stop and move signals $\tau^{A}$ and $\tau^{B}$, whereas an opponent can only receive these signals but not influence the partners with its own output bit. This is the essential mechanism which allows synchronization but prohibits learning. Numerical [9] as well as analytical [10] calculations of the dynamic process show that the partners can synchronize in a short time whereas an opponent needs a much longer time to lock into the partners.

This observation holds for an observer who uses the same algorithm (13) as the two partners $A$ and $B$. Note that the observer knows 1. the algorithm of $A$ and $B, 2$. the input vectors $\underline{x}_{1}, \underline{x}_{2}, \underline{x}_{3}$ at each time step and 3 . the output bits $\tau^{A}$ and $\tau^{B}$ at each time step. Nevertheless, he does not succeed in synchronizing with $A$ and $B$ within the communication period.

Since for each run the two partners draw random initial weights and since the input vectors are random, one obtains a distribution of synchronization times as shown in Fig. 4 for $N=100$ and $L=3$. The average value of this distribution is shown as a function of system size $N$ in Fig. F. Even an infinitely large network needs only a finite number of exchanged bits - about 

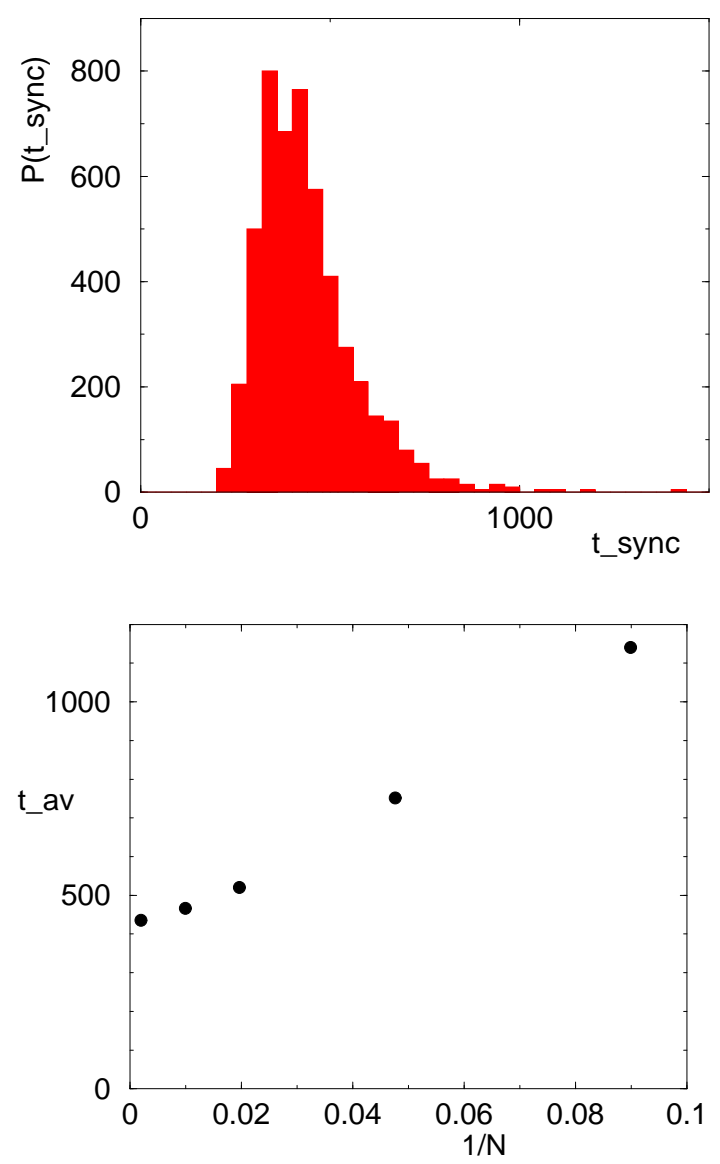

Fig. 4. Distribution of synchronization time for $N=$ $100, L=3$.

Fig. 5. Average synchronization time as a function of inverse system size.

400 in this case - to synchronize, in agreement with the analytical calculation for $N \rightarrow \infty$.

If the communication continues after synchronization, an opponent has a chance to lock into the moving weights of $A$ and $B$. Fig. 6 shows the distribution of the ratio between the synchronization time of $A$ and $B$ and the learning time of the opponent. In our simulations, for $N=100$, this ratio never exceeded the value $r=0.1$, and the average learning time is about 50000 time steps, much larger than the synchronization time. Hence, the two partners can take their weights $\underline{w}_{i}^{A}(t)=\underline{w}_{i}^{B}(t)$ at a time step $t$ where synchronization most probably occurred as a common secret key. Synchronization of neural networks can be used as a key exchange protocol over a public channel. 


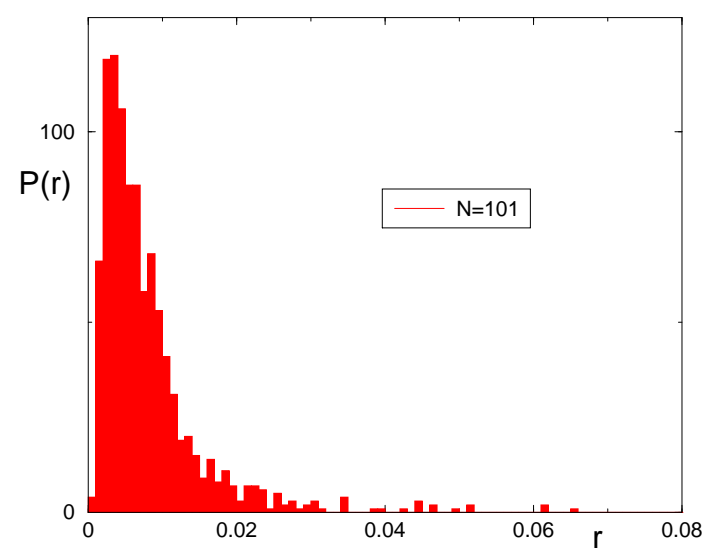

Fig. 6. Distribution of the ratio of synchronization time between networks $\mathrm{A}$ and $\mathrm{B}$ to the learning time of an attacker E.

\section{Conclusions}

Interacting neural networks have been calculated analytically. At each training step two networks receive a common random input vector and learn their mutual output bits. A new phenomenon has been observed: Synchronization by mutual learning. If the learning rate $\eta$ is large enough, and if the weight vectors keep normalized, then the two networks relax to an antiparallel orientation. Their weight vectors still move like a random walk on a hypersphere, but each network has complete knowledge about its partner.

It has been shown how this phenomenon can be used for cryptography. The two partners can agree on a common secret key over a public channel. An opponent who is recording the public exchange of training examples cannot obtain full information about the secrete key used for encryption.

This works if the two partners use multilayer networks, parity machines. The opponent has all the informations (except the initial weight vectors) of the two partners and uses the same algorithms. Nevertheless he does not synchronize.

This phenomenon may be used as a key exchange protocol. The two partners select secret initial weight vectors, agree on a public sequence of input vectors and exchange public bits. After a few steps they have identical weight vectors which are used for a secret encryption key. For each communication they agree on a new secret key, without having stored any secret information before. In contrast to number theoretical methods the networks are very fast; essentially they are linear filters, the complexity to generate a key of length $N$ scales with $N$ (for sequential update of the weights).

Of course, one cannot rule out that algorithms for the opponent may be constructed which find the key in much shorter time. In fact, ensembles of opponents have a better chance to synchronize. In addition, one can show that, given the information of the opponent, the key is uniquely determined, and, given the sequence of inputs, the number of keys is huge but finite, even in the 
limit $N \rightarrow \infty$ [11]. These may be good news for a possible attacker. However, recently we have found advanced algorithms for synchronization, too. Such variations are subjects of active research, and future will show whether the security of neural network cryptography can compete with number theoretical methods.

Acknowledgments: This work profitted from enjoyable collaborations with Richard Metzler and Michal Rosen-Zvi. We thank the German Israel Science Foundation (GIF) and the Minerva Center of the Bar-Ilan University for support.

\section{References}

1. J. Hertz, A. Krogh, and R. G. Palmer: Introduction to the Theory of Neural Computation, (Addison Wesley, Redwood City, 1991)

2. A. Engel, and C. Van den Broeck: Statistical Mechanics of Learning, (Cambridge University Press, 2001)

3. M. Biehl and N. Caticha: Statistical Mechanics of On-line Learning and Generalization, The Handbook of Brain Theory and Neural Networks, ed. by M. A. Arbib (MIT Press, Berlin 2001)

4. E. Eisenstein and I. Kanter and D.A. Kessler and W. Kinzel, Phys. Rev. Lett. 74, 6-9 (1995)

5. I. Kanter, D.A. Kessler, A. Priel and E. Eisenstein, Phys. Rev. Lett. 75, 26142617 (1995); L. Ein-Dor and I. Kanter, Phys. Rev. E 57, 6564 (1998); M. Schröder and W. Kinzel, J. Phys. A 31, 9131-9147 (1998); A. Priel and I. Kanter, Europhys. Lett. (2000)

6. R. Metzler and W. Kinzel and I. Kanter, Phys. Rev. E 62, 2555 (2000)

7. D. R. Stinson, Cryptography: Theory and Practice (CRC Press 1995)

8. R. Metzler, W. Kinzel, L. Ein-Dor and I. Kanter, Phys. Rev. E 63, 056126 (2001)

9. I. Kanter, W. Kinzel and E. Kanter, Europhys. Lett. 57, 141-147 (2002)

10. M. Rosen-Zvi, I. Kanter and W. Kinzel, cond-mat/0202350 (2002)

11. R. Urbanczik, private communication 